\title{
Interlayer exchange coupling in digital magnetic alloys
}

\author{
V. N. Men'shov, ${ }^{1,2}$ V. V. Tugushev, ${ }^{1,2}$ P. M. Echenique,,${ }^{1,3}$ S. Caprara, ${ }^{4}$ and E. V. Chulkov ${ }^{1,3}$ \\ ${ }^{1}$ Donostia International Physics Center (DIPC), P. de Manuel Lardizabal 4, 20018, San Sebastián, Basque Country, Spain \\ ${ }^{2}$ RRC Kurchatov Institute, Kurchatov Square 1, 123182 Moscow, Russia \\ ${ }^{3}$ Departamento de Física de Materiales, Facultad de Ciencias Químicas, UPV/EHU and Centro Mixto CSIC-UPV/EHU, Apartado 1072, \\ 20080 San Sebastián, Basque Country, Spain \\ ${ }^{4}$ Dipartimento di Fisica, Universita di Roma "La Sapienza" and Instituto Nazionale per la Fisica della Materia, SMC \\ and UDR di Roma 1, Piazalle Aldo Moro 2, 00185 Rome, Italy
}

(Received 28 April 2008; revised manuscript received 30 June 2008; published 29 July 2008)

\begin{abstract}
We discuss possible mechanisms for indirect exchange between ferromagnetic $\delta$ layers of transition metal inserted into a semiconducting host, taking into account the role of carrier confinement at these layers. We show that the Ruderman-Kittel-Kasuya-Yoshida mechanism is not the ultimate explanation for an interlayer interaction and an efficient interlayer coupling can be mediated by the undoped semiconducting spacer due to virtual excitations across the energy threshold. We emphasize the important role of quasi-two-dimensional spin-polarized states inside the bulk energy gap, which are caused by the confinement and the exchange scattering of itinerant electrons by the $\delta$ layers. Quasiparticle excitations from these states to the band edge of the spacer contribute to the interlayer coupling even for a wide gap semiconducting spacer. Our analysis shows that the related exchange integral can change its sign at some "critical" spacer thickness, i.e., a ferromagnetic coupling mechanism is active at short distance between $\delta$ layers and an antiferromagnetic coupling mechanism is active at large distance. Taking into account the effects of crystal symmetry, we also obtain the expression for the interband coupling energy in the case of both direct- and indirect-gap spacers. We show that the carrier confinement gives rise to a renormalization of the intensity of excitations through the band gap. The interband coupling decays exponentially with the distance between the $\delta$ layers and is strongly determined by the electron structure of the host. The estimates of the interlayer interaction parameters across $\mathrm{Si}, \mathrm{Ge}$, and $\mathrm{GaAs}$ spacers are presented. The combination of two mechanisms (confinement-mediated exchange and interband exchange) mainly determines the behavior of the interlayer coupling in the digital magnetic alloys with undoped spacer.
\end{abstract}

DOI: 10.1103/PhysRevB.78.024438

PACS number(s): 73.40.Sx, 75.70.-i

\section{INTRODUCTION}

A unique combination of properties makes hybrid ferromagnetic metal/semiconductor (FM/SC) layered nanostructures very attractive for various spintronic applications. ${ }^{1}$ These layered materials allow for locally high concentrations of magnetic ions, significantly exceeding the solubility limit in the bulk diluted magnetic semiconductors (DMS). The strength of the exchange interaction between magnetic moments and band electrons of the spacer at interfaces in FM/SC nanostructures is of orders of magnitude larger than in the bulk of DMS. Hence, the control of magnetic and magnetotransport characteristics in FM/SC nanostructures would be more efficient than in DMS quantum wells.

Molecular beam epitaxy techniques with monolayer precision have reached a high degree of accuracy so that it is now possible to prepare FM/SC digital magnetic alloys (DMA), in which the monolayers or submonolayers of transition metals, embedded into SC host by means of selective doping, form the so-called FM $\delta$ layers. ${ }^{1}$ By engineering the composition of the FM and of the SC, as well as the distance between FM $\delta$ layers, one can vary the band spectrum and factors influencing magnetic order in these materials.

Among DMA, the structures based on III-V SC compounds have been most extensively studied. Thanks to wellestablished growth expertise, some interesting technological procedures have recently been devised. One example is the digital heterostructure GaAs/MnAs grown by alternate deposition of nanolayers of GaAs (SC) and MnAs (FM). ${ }^{2,3}$ Digital alloys (GaAs, $\mathrm{GaSb}) / \mathrm{Mn}$ have been grown by periodically embedding submonolayers of magnetic atoms ( $\mathrm{Mn}$ ) into the $\mathrm{SC}$ host (GaAs or GaSb). ${ }^{4-6}$ Note also the original method of $\delta$ doping of Mn atoms into the SC (GaAs)-based heterostructures, successively achieved in Refs. 7 and 8. Despite the relevance and special interest for integration into mature silicon technologies for spintronic application, rather little attention has been paid to DMA based on the group IV SC (mainly $\mathrm{Si}$ and Ge). There exist, nevertheless, serious evidences for FM ordering in DMS of type Ge:Mn (Refs. 9 and 10) and Si:Mn (Refs. 11 and 12) as well as in multilayers of type $\mathrm{Fe} / \mathrm{Si}^{13,14}$

On the other hand, $a b$ initio electron structure calculations have been performed for DMA Mn/GaAs, ${ }^{15-18} \mathrm{Mn} / \mathrm{Ge},{ }^{19,20}$ and $\mathrm{Mn} / \mathrm{Si}^{21,22}$ In these calculations, the model of a sequence of $\mathrm{Mn}$ monolayers, periodic along the (001) direction and separated by several monolayers of the SC host, has been used. It has been found that (i) FM order in the Mn layer is energetically more favored than antiferromagnetic (AFM) order; (ii) the strong Coulomb and exchange interactions of carriers with Mn ions lead to carrier confinement at the FM layers; and (iii) the electron spectrum is half-metallic and has strongly spin-polarized two-dimensional energy bands.

The results of numeric calculations demonstrate an important effect of $(s, p)-d$ hybridization and charge redistribution between FM monolayers and SC host on the DMA band 
structure. The electron spectrum of DMA has a very complex character, containing both strongly correlated (Hubbard-like) narrow bands and weakly correlated wide bands. The FM order inside each metal monolayer leads to spin splitting of the electron states, thus lowering the total energy of the system and forming the spin-polarized bands. The description of an intralayer FM order due to strong correlations in the narrow electron bands, associated with a half-metallic state, is similar to that of the conventional model of itinerant ferromagnetism in transition metals and their alloys. ${ }^{23}$

Following the results of numerical calculations, ${ }^{15-22}$ we suppose in this work that FM order exists inside each $\delta$ layer and concentrate on a question: what is the mechanism of an interlayer exchange coupling (IEC) in DMA systems? In principle, $a b$ initio calculations might simultaneously describe both the intralayer and interlayer magnetic orders in the system. However, to obtain sufficient numerical accuracy, these studies have really been restricted to relatively small spacer thicknesses since it was necessary to calculate very small deviations in total energy between different magnetic configurations, for instance, between parallel and antiparallel alignments of moments at neighboring FM layers ( $a$ priori, the noncollinear configurations cannot be also excluded either). Moreover, the modern epitaxial technology produces nanostructures that are too far from the ideal ones used in first principle calculations. In real DMA, nominal monolayers or submonolayers appear rather as alloying regions, smeared along the growth direction.

To our knowledge, the origin of IEC in DMA has not been sufficiently studied so far. The main problem is to reveal the groups of electron states that dominate IEC. Several scenarios may be envisioned. In principle, the Fermi-level position of the system strongly depends on the charge redistribution between the SC spacer and FM $\delta$ layers, and can be shifted into the conduction or valence band of the spacer. Then, the usual Ruderman-Kittel-Kasuya-Yoshida (RKKY)like exchange mechanism via free carriers (real electron-hole excitations in the vicinity of the Fermi level) dominates the interaction between FM $\delta$ layers. This interaction has a behavior that oscillates with the distance, as in conventional metals or degenerate semiconductors. On the other hand, if the Fermi level is located within the energy gap of the spacer, the IEC, determined by virtual electron-hole excitations through the energy threshold, acquires an exponential decay at large distances. As a rule, this energy threshold is simply associated with the energy gap of the SC spacer although in some layered structures it might be less than the gap thanks to the presence of the spin-split interface states inside the fundamental gap. As it is shown in several works (see, for example, Ref. 24$)$, the $(s, p)-d$ hybridization occurs to provide a strong superexchange contribution to IEC when the electron density of states (DOS) exhibits a peak-shaped feature close to the Fermi level. If the DOS peak is rather large, virtual transition between the peak and the conduction (valence) band edge could dominate IEC. This situation probably takes place in the well-known multilayered system $\mathrm{Fe} / \mathrm{Fe}_{1-x} \mathrm{Si}_{x}(001)$ with $x>0.5$, which has been described in the framework of the superexchange model in Ref. 25.

In view of the complexity of the problem, and since IEC is highly sensitive to a choice of type and thickness of both the SC spacer and the FM layer, we would like to propose comprehensible qualitative scheme describing this phenomenon.

The paper is organized as follows. In Sec. II we briefly discuss a simple model of DMA. In Sec. III we develop a formalism describing two-dimensional electron states in a system composed of two FM $\delta$ layers separated by a SC spacer. In Sec. IV the IEC energies of such system are calculated for a FM and an AFM alignment of the moments of adjacent $\delta$ layers. In Sec. V we analyze the interband contribution into IEC for DMA based on IV and III-V SCs. In Sec. VI we summarize our results and discuss some unsolved problems. Finally, in the Appendix we give rough estimates for the characteristic lengths of IEC, based on electron structure calculations.

\section{MODEL}

In this section we propose a simple model allowing us to describe the dependence of the IEC integral on the SC spacer thickness. Let us consider the fragment of DMA consisting of two parallel FM $\delta$ layers embedded in a crystalline SC host. The Hamiltonian $H$ of the SC electron states, which are spin polarized by the presence of the FM $\delta$ layers, may be written in the following form:

$$
\begin{gathered}
H=H_{0}+V(+l)+V(-l), \\
H_{0}=\sum_{n \mathbf{k} \alpha} \varepsilon_{n \mathbf{k}} a_{n \mathbf{k} \alpha}^{+} a_{n \mathbf{k} \alpha},
\end{gathered}
$$

and

$$
\begin{aligned}
V( \pm l)= & -\sum_{n \mathbf{k}} \sum_{n^{\prime} \mathbf{k}^{\prime}} \sum_{\alpha, \beta} a_{n \mathbf{k} \alpha}^{+}\left\{U_{n \mathbf{k}, n^{\prime} \mathbf{k}^{\prime}}( \pm l)+J_{n \mathbf{k}, n^{\prime} \mathbf{k}^{\prime}}( \pm l)\right. \\
& \left.\times[\boldsymbol{\sigma} \cdot \mathbf{M}( \pm l)]_{\alpha \beta}\right\} a_{n^{\prime} \mathbf{k}^{\prime} \beta} \exp \left[i\left(k_{z}-k_{z}^{\prime}\right)( \pm l)\right] .
\end{aligned}
$$

Here, $H_{0}$ is the Hamiltonian of a bulk SC, $a^{+}(a)$ is the creation (annihilation) operator, $\varepsilon_{n \mathbf{k}}$ are the quasiparticle energies, $\left(\mathbf{k}, \mathbf{k}^{\prime}\right),\left(n, n^{\prime}\right)$, and $(\alpha, \beta)$ are momenta, band, and spin indices of the Bloch states, respectively. The Hamiltonian $V( \pm l)$ describes the interaction of the SC host with the FM $\delta$ layers. Denoting $z$ as the coordinate along the heterostructure growth direction, we designed these layers to be infinitesimally thin planes placed at $z= \pm l$. Thus, the system is uniform and infinite in the transverse directions $\mathbf{x}$ and y. As it can be seen from Eq. (3), the metal layers are assumed to excite both charge and spin densities in the SC. In this work, we treat the spin density of the layers classically so the vectors $\mathbf{M}( \pm l)$ represent the mean magnetization of the "right" $(+l)$ and "left" $(-l)$ layers, the distance between two FM planes is $2 l$, and $\boldsymbol{\sigma}$ is a vector composed of the Pauli matrices. We assume that the vectors $\mathbf{M}(+l)$ and $\mathbf{M}(-l)$ lay in the $(x, y)$ plane. The matrix elements $U_{n \mathbf{k}, n^{\prime} \mathbf{k}^{\prime}}( \pm l)$ and $J_{n \mathbf{k}, n^{\prime} \mathbf{k}^{\prime}}( \pm l)$ describe the intensities of the potential and exchange scatterings by the $\delta$ layers, respectively. It is assumed that the values of the matrix elements are independent of the position $( \pm l): M(+l)=M(-l)=M, U_{n \mathbf{k}, n^{\prime} \mathbf{k}^{\prime}}(+l)=U_{n \mathbf{k}, n^{\prime} \mathbf{k}^{\prime}}(-l)$ $=U_{n \mathbf{k}, n^{\prime} \mathbf{k}^{\prime}}$, and $J_{n \mathbf{k}, n^{\prime} \mathbf{k}^{\prime}}(+l)=J_{n \mathbf{k}, n^{\prime} \mathbf{k}^{\prime}}(-l)=J_{n \mathbf{k}, n^{\prime} \mathbf{k}^{\prime}}$. Notice also 
that the interaction is local in the $\mathbf{z}$ direction and strictly periodic in the $(x, y)$ plane so that the matrix elements $U_{n \mathbf{k}, n^{\prime} \mathbf{k}^{\prime}}=U_{n, n^{\prime}}\left(\mathbf{k}_{\|}-\mathbf{k}_{\|}^{\prime}\right)$ and $J_{n \mathbf{k}, n^{\prime} \mathbf{k}^{\prime}}=J_{n, n^{\prime}}\left(\mathbf{k}_{\|}-\mathbf{k}_{\|}^{\prime}\right)$ depend only on the difference of the longitudinal components of momenta $\left(\mathbf{k}, \mathbf{k}^{\prime}\right)$ from the first Brillouin zone of the SC host.

In DMA, the valence chemical bonding between ions of transition metal and nearest neighboring ions of SC, as well as the corresponding electronic states at the FM $\delta$ layer, is highly complex. In the framework of our approach, we simply treat the FM $\delta$ layer in the host as a source of scattering entailed in the potential $U( \pm l)$ and the exchange $J( \pm l)$ components in the Hamiltonian (3). Such an assumption allows us to construct a physically transparent analytical scheme. It is implied that band carriers moving along the $\mathbf{z}$ axis are strongly scattered by the two FM $\delta$ layers while they keep a free propagation along the interface $(x, y)$ plane. Of course, electrons of the SC host are spin polarized in the vicinity of the FM $\delta$ layer. Moreover, the magnetic configuration of the system depends self-consistently on the rearrangement of the electron spectrum of the SC host, caused by both the potential $\left(U_{n \mathbf{k}, n^{\prime} \mathbf{k}^{\prime}}\right)$ and exchange $\left(J_{n \mathbf{k}, n^{\prime} \mathbf{k}^{\prime}}\right)$ components of the scattering [Eq. (3)]. In turn, the spin density distribution of carriers determines IEC between the FM $\delta$ layers through the $\mathrm{SC}$ spacer.

\section{SPIN-POLARIZED ELECTRON STATES INDUCED BY FM $\delta$ LAYERS}

We start our analysis by adopting a single-band description of the electron structure of the system: $n=n^{\prime}=1$ and $\varepsilon_{1 \mathbf{k}}=\varepsilon(\mathbf{k})$, where $\mathbf{k}=\left(k_{x}, k_{y}, k_{z}\right)$ is the momentum measured from a relevant point of the first Brillouin zone of the SC. The Green function of the Hamiltonian (1)-(3) is given by

$$
G_{\alpha \beta}\left(\mathbf{k}, \mathbf{k}^{\prime}, \omega\right)=\delta_{\mathbf{k}, \mathbf{k}^{\prime}} \delta_{\alpha \beta} G^{0}(\mathbf{k}, \omega)+\delta G_{\alpha \beta}\left(\mathbf{k}, \mathbf{k}^{\prime}, \omega\right),
$$

where $G^{0}(\mathbf{k}, \omega)=[\omega-\varepsilon(\mathbf{k})]^{-1}$ is the Green function of bulk $\mathrm{SC}$ host. The additional term

$$
\delta G_{\alpha \beta}\left(\mathbf{k}, \mathbf{k}^{\prime}, \omega\right)=\delta_{\mathbf{k}_{\|}, \mathbf{k}_{\|}^{\prime}} G^{0}(\mathbf{k}, \omega) T_{\alpha \beta}\left(\mathbf{k}, \mathbf{k}^{\prime}, \omega\right) G^{0}\left(\mathbf{k}^{\prime}, \omega\right)
$$

appears as a result of the interaction $V( \pm l)[$ Eq. (3)] with the $\delta$ layers; here the function

$$
\begin{aligned}
T_{\alpha \beta}\left(\mathbf{k}, \mathbf{k}^{\prime}, \omega\right)= & \left\{Q_{\alpha \beta}^{+}\left(\mathbf{k}_{\|}, \omega\right) \exp \left[i\left(k_{z}-k_{z}^{\prime}\right) l\right]\right. \\
& +Q_{\alpha \beta}^{-}\left(\mathbf{k}_{\|}, \omega\right) \exp \left[-i\left(k_{z}-k_{z}^{\prime}\right) l\right] \\
& +P_{\alpha \beta}^{+}\left(\mathbf{k}_{\|}, \omega\right) \exp \left[i\left(k_{z}+k_{z}^{\prime}\right) l\right] \\
& \left.+P_{\alpha \beta}^{-}\left(\mathbf{k}_{\|}, \omega\right) \exp \left[-i\left(k_{z}+k_{z}^{\prime}\right) l\right]\right\}\left[\Delta\left(\mathbf{k}_{\|}, \omega\right)\right]^{-1}
\end{aligned}
$$

is the full $t$ matrix, which represents the multiple scattering of carriers by the defect of two parallel $\delta$ layers inserted in the SC medium. The following notations are adopted:

$$
\begin{aligned}
\Delta= & D^{2}-2 g^{2}\left[K^{2}+J^{2} M^{2}\left(\mathbf{n}_{+} \cdot \mathbf{n}_{-}\right)\right]+g^{4}\left(U^{2}-J^{2} M^{2}\right)^{2}, \\
Q_{\alpha \beta}^{ \pm}= & D\left[K \delta_{\alpha \beta}+J M\left(\boldsymbol{\sigma} \cdot \mathbf{n}_{ \pm}\right)_{\alpha \beta}\right]-g^{2}\left(U^{2}-J^{2} M^{2}\right)\left[K \delta_{\alpha \beta}\right. \\
& \left.-J M\left(\boldsymbol{\sigma} \cdot \mathbf{n}_{ \pm}\right)_{\alpha \beta}\right],
\end{aligned}
$$

$$
\begin{aligned}
P_{\alpha \beta}^{ \pm}= & g\left[K^{2}+J^{2} M^{2}\left(\mathbf{n}_{+} \cdot \mathbf{n}_{-}\right)\right] \delta_{\alpha \beta}-g^{3}\left(U^{2}-J^{2} M^{2}\right)^{2} \delta_{\alpha \beta} \\
& +g K J M\left[\boldsymbol{\sigma} \cdot\left(\mathbf{n}_{+}+\mathbf{n}_{-}\right)\right]_{\alpha \beta}+i g J^{2} M^{2}\left[\boldsymbol{\sigma} \cdot\left(\mathbf{n}_{+} \times \mathbf{n}_{-}\right)\right]_{\alpha \beta},
\end{aligned}
$$

$$
\begin{aligned}
g_{0} & =g_{0}\left(\mathbf{k}_{\|}, \omega\right)=G^{0}\left(l, l, \mathbf{k}_{\|}, \omega\right)=G^{0}\left(-l,-l, \mathbf{k}_{\|}, \omega\right) \\
& =G^{0}\left(0,0, \mathbf{k}_{\|}, \omega\right)=\sum_{k_{z}} G^{0}(\mathbf{k}, \omega)=\int \frac{a_{z} d k_{z}}{2 \pi} G^{0}(\mathbf{k}, \omega),
\end{aligned}
$$

and

$$
\begin{aligned}
g & =g\left(\mathbf{k}_{\|}, \omega\right)=G^{0}\left(l,-l, \mathbf{k}_{\|}, \omega\right)=G^{0}\left(-l, l, \mathbf{k}_{\|}, \omega\right) \\
& =\sum_{k_{z}} G^{0}(\mathbf{k}, \omega) \exp \left(2 i k_{z} l\right)=\int \frac{a_{z} d k_{z}}{2 \pi} G^{0}(\mathbf{k}, \omega) \exp \left(2 i k_{z} l\right),
\end{aligned}
$$

$$
\mathbf{M}( \pm l)=M \mathbf{n}_{ \pm},
$$

where $\mathbf{k}_{\|}=\left(k_{x}, k_{y}\right)$ is the $(x, y)$ component of momentum parallel to the interface, $\mathbf{k}=\left(\mathbf{k}_{\|}, k_{z}\right)$, and $a_{z}$ is the lattice spacing of the SC along the $\mathbf{z}$ axis. We take the intraband matrix elements at the band extremum point, $U=U_{1,1}(0)$ and $J$ $=J_{1,1}(0)$, in which all the other matrix elements are assumed to be negligibly small. Note also that the above parameters are linked by the relation

$$
D=1-(U+K) g_{0} .
$$

The poles of the scattering matrix [Eq. (6)] give the electron spectrum of the system. It should be noted that the determinant $\Delta(\omega)$ depends parametrically on both the mutual orientation of the magnetic moments of the inserted layers, $\left(\mathbf{n}_{+} \cdot \mathbf{n}_{-}\right)$, and on the distance between them, $2 l$. Since we are only interested in the case when the Fermi level lies inside the SC band gap, only the solutions of the equation $\Delta(\omega)$ $=0$ with negative energies, $\omega<0$, are considered below.

If the interlayer distance tends to infinity, $l \rightarrow \infty$ (accordingly, $g \rightarrow 0$ ), the single $\delta$ layer situation occurs. In the system with a short-range attractive potential, $U<0$, there is always a bound state for a particle moving perpendicularly to the $\delta$ layer. The twofold spin degeneration of this state is removed due to the presence of the magnetic layer. In the case of a single plane with $J M \neq 0$, the spectrum is simply given by $D(\omega)=0$, i.e.,

$$
g_{0}^{-1}\left(\mathbf{k}_{\|}, \omega\right)=U \pm J M .
$$

We shall assume in what follows that the condition $|U|$ $>|J M|$ is fulfilled.

In the case of two parallel FM layers, each state inherent to a single layer is split into a bounding and an antibounding state. The splitting is proportional to the overlap integral of wave functions localized at the different planes, which is the value $\left(g / g_{0}\right)^{2}$. As a consequence of quantization of the trans- 
verse (relative to the layer plane) propagation of carriers, four two-dimensional subbands are formed inside the gap of the SC host, $\omega=\omega_{i}\left(\mathbf{k}_{\|}\right)$, where $i=1,2,3$, and $4, i$ is the subband index.

The electron DOS variation per spin direction caused by the $\delta$ layer, taking into account Eq. (5), is given by

$$
\delta N_{\alpha}(\omega)=-\frac{2}{\pi} \operatorname{Im} \sum_{\mathbf{k}_{\|}} T_{\alpha \alpha}(\mathbf{k}, \mathbf{k}, \omega)\left[G^{0}(\mathbf{k}, \omega)\right]^{2} .
$$

For the sake of simplicity, we express the electron spectrum in the form: $\varepsilon(\mathbf{k})=\varepsilon_{\|}\left(\mathbf{k}_{\|}\right)+\varepsilon_{\perp}\left(k_{z}\right)$, and write the full variation of the DOS as

$$
\begin{aligned}
\delta N(\omega)= & \sum_{\alpha} \delta N_{\alpha}(\omega) \\
= & \frac{4}{\pi \Delta} \operatorname{Im} \int d \varepsilon_{\|} N_{2}\left(\varepsilon_{\|}\right)\left\{K\left[D-g\left(U^{2}-J^{2} M^{2}\right)\right] \frac{\partial g_{0}}{\partial \omega}\right. \\
& \left.+g\left[K^{2}+J^{2} M^{2}\left(\mathbf{n}_{+} \cdot \mathbf{n}_{-}\right) g^{2}\left(U^{2}-J^{2} M^{2}\right)^{2}\right] \frac{\partial g}{\partial \omega}\right\},
\end{aligned}
$$

where the two-dimensional DOS is introduced in the standard way, promoting wave-vector sums to energy integrals,

$$
\sum_{k_{\|}} \rightarrow \int \frac{a_{\|}^{2} d \mathbf{k}_{\|}}{(2 \pi)^{2}} \rightarrow \int d \varepsilon_{\|} N_{2}\left(\varepsilon_{\|}\right),
$$

where the length $a_{\|}$is the lattice period of the SC in the plane $(x, y)$. In the following, we shall omit the "transverse" index $\perp$, i.e., assign $\varepsilon_{\perp}=\varepsilon$. After some manipulations, one reduces Eq. (16) to the following expression:

$$
\delta N(\omega)=\sum_{i} \int d \varepsilon_{\|} N_{2}\left(\varepsilon_{\|}\right) \delta\left(\omega-\omega_{i}\left(\mathbf{k}_{\|}\right)\right),
$$

where

$$
\omega_{i}\left(\mathbf{k}_{\|}\right)=\varepsilon_{i}+\varepsilon_{\|}\left(\mathbf{k}_{\|}\right)
$$

is the $i$ th branch of the electron spectrum, which has been defined above as a solution of the equation $\Delta(\omega)=0$ and $\delta(\omega)$ is delta function. Here we consider electron states near the conduction-band bottom, which we take as the zero energy. In this case, $\varepsilon_{i}<0$. Of course, one could change the signs accordingly in order to depict states near the top of the valence band. The value $\varepsilon_{i}$ determines the position of a minimum of the $i$ th subband with respect to the edge of the threedimensional band of the SC host, i.e., the depth of the state inside the band gap localized in the $\mathbf{z}$ direction.

In the following, in order to carry out concrete calculations, we use for the band electron energy the parabolic form $\varepsilon_{1 \mathbf{k}}=\varepsilon(\mathbf{k})=\frac{\mathbf{k}_{\|}^{2}}{2 m_{\|}}+\frac{k_{z}^{2}}{2 m}$, where $m_{\|}$and $m$ are the "longitudinal" and transverse effective masses near the bottom of the conduction band, respectively. On the one hand, the effectivemass approach is quite accurate because actual quasiparticle energies are small in comparison to the width of the conducting band, $W(|\omega| \ll W)$; on the other hand, it allows us to simplify the calculations. Indeed, the functions $g_{0}$ [Eq. (12)] and $g$ [Eq. (13)] take the fairly simple forms:

$$
g_{0}=-a_{z} \sqrt{\frac{m}{2(-\varepsilon)}}
$$

and

$$
g=g_{0} \exp [-2 l \sqrt{2 m(-\varepsilon)}],
$$

where $\varepsilon=\omega-\varepsilon_{\|}$is the energy of an electron moving normal to the interface. Inserting the values $g_{0}[\mathrm{Eq} .(19)]$ and $g[\mathrm{Eq}$. (20)] into Eq. (7), one may cast the equation $\Delta(\omega)=0$ in the form

$$
\begin{aligned}
(\sqrt{E}-1)^{4}-2(A+B)(\sqrt{E}-1)^{2}-4 A B(\sqrt{E}-1) & \\
& -2 A B\left(\mathbf{n}_{+} \cdot \mathbf{n}_{-}\right) E+(A+B-A B)^{2}-2 A B=0,
\end{aligned}
$$

where we introduced the dimensionless parameters

$$
\begin{gathered}
E=\frac{2|\varepsilon|}{m a_{z}^{2} U^{2}}, \\
A=A(\omega)=\left(\frac{g}{g_{0}}\right)^{2}=\exp (-L \sqrt{E}), \\
B=\left(\frac{J M}{U}\right)^{2}, \\
L=4 \operatorname{lm} a_{z}|U| .
\end{gathered}
$$

We notice several characteristic features of the solutions of Eq. (21), i.e., the discrete energies $\varepsilon=\varepsilon_{i}$, for the DMA structure considered here.

If $\left(\mathbf{n}_{+} \cdot \mathbf{n}_{-}\right) \neq-1$, Eq. (21) has four real solutions $E_{i}$ (we assume that $\left.E_{1}>E_{2}>E_{3}>E_{4}\right)$ at large distances $L>L_{4}$, three solutions $\left(E_{1}>E_{2}>E_{3}\right)$ in interval $L_{3}<L<L_{4}$, and two solutions $\left(E_{1}>E_{2}\right)$ in the region $L<L_{3}$. In this manner, two out of four states move from the band gap to the continuum, i.e., they become resonant states when $L=L_{3,4}$, where

$$
L_{4,3}=\frac{2 \pm \sqrt{2 B\left(1+\mathbf{n}_{+} \cdot \mathbf{n}_{-}\right)}}{1-B} .
$$

At arbitrary alignment of the magnetic moments, the asymptotic behavior of $E(L)$ at $L \gg 1$ and $L>L_{4}$ can be expressed as

$$
\sqrt{E_{1,2}(L)}=\sqrt{E_{0}^{(+)}}\left[1 \pm \sqrt{\frac{1}{2} A^{(+)}(L)\left(1+\mathbf{n}_{+} \cdot \mathbf{n}_{-}\right)}+\Theta^{(+)}(L)\right],
$$

and

$$
\sqrt{E_{3,4}(L)}=\sqrt{E_{0}^{(-)}}\left[1 \pm \sqrt{\frac{1}{2} A^{(-)}(L)\left(1+\mathbf{n}_{+} \cdot \mathbf{n}_{-}\right)}+\Theta^{(-)}(L)\right],
$$

where 


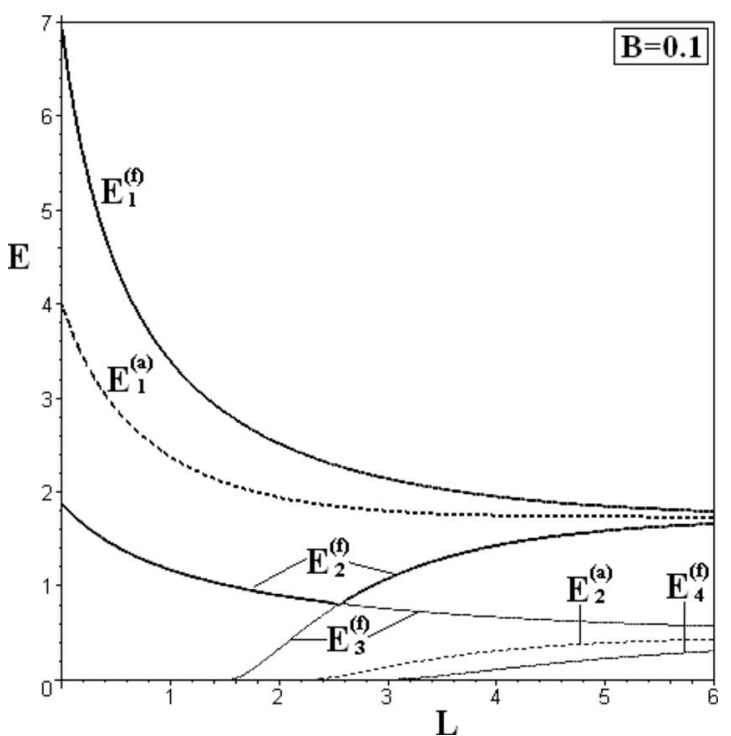

FIG. 1. The variation of the subband-edge position $E_{i}^{(f)}(L)$ and $E_{i}^{(a)}(L)$ as a function of the spacer thickness $L$ at $B=0.1$. The energy states $E_{1}^{(f)}(L), E_{2}^{(f)}(L)$, and $E_{1}^{(a)}(L)$ are depicted by solid bold and dashed bold lines, respectively; the energy states $E_{3}^{(f)}(L), E_{4}^{(f)}(L)$, and $E_{2}^{(a)}(L)$ are depicted by solid thin and dashed thin lines, respectively.

$$
\begin{aligned}
& \Theta^{( \pm)}(L)= \pm \frac{A^{( \pm)}(L)}{4}\left[\frac{\left(1-\mathbf{n}_{+} \cdot \mathbf{n}_{-}\right) \sqrt{E_{0}^{(\mp)}}}{\sqrt{B}}\right. \\
&\left.-L\left(1+\mathbf{n}_{+} \cdot \mathbf{n}_{-}\right) \sqrt{E_{0}^{( \pm)}}\right] \\
& A^{( \pm)}(L)=\exp \left(-L \sqrt{E_{0}^{( \pm)}}\right) .
\end{aligned}
$$

The values $E_{0}^{( \pm)}=(1 \pm \sqrt{B})^{2}$ are the dimensionless energies of the localized electron states in the case of a single $\delta$ layer in the host [see Eq. (14)].

In the case of FM alignment of the moments, $\mathbf{n}_{+} \cdot \mathbf{n}_{-}=1$, we denote the solutions of Eq. (21) as $E_{i}^{(f)}, i=1,2,3$, and 4. At some intermediate point of the interval $\left(L_{3}, L_{4}\right)$, the terms $E_{2}^{(f)}$ and $E_{3}^{(f)}$ become degenerate, i.e., there is a level crossing.

Figure 1 shows the typical behavior of the levels $\varepsilon_{i}$ as functions of the interlayer distance $2 l$ (actually, we plot the dimensionless parameters $E_{i}$ vs $L$ for a collinear orientations of the moments) and $B=0.1$.

It is worthwhile noting that for the antiparallel (AFM) configuration of the magnetic moments of the two planes, $\mathbf{n}_{+} \cdot \mathbf{n}_{-}=-1$, when the total magnetization of the system is equal to zero, the spin splitting of the localized states disappears and one is left with a pair of twofold-degenerate bands, $\omega_{i}^{(a)}\left(\mathbf{k}_{\|}\right)=\varepsilon_{i}^{(a)}+\varepsilon_{\|}\left(\mathbf{k}_{\|}\right), i=1$ and 2 ; the energy minima of which obey the equation:

$$
(\sqrt{E}-1)^{2}+A B-A-B=0 .
$$

We denote the solutions of Eq. (26) using the symbols $E_{1,2}^{(a)}$. The solution $E_{2}^{(a)}$ exists under the condition $L>2 /(1-B)$ and $E_{1}^{(a)}>E_{2}^{(a)}$. The asymptotic behavior of the function $E^{(a)}(L)$, when $L \gg 1$, is described by the relation

$$
\sqrt{E_{1,2}^{(a)}(L)}=\sqrt{E_{0}^{( \pm)}} \pm \frac{1-B}{2 \sqrt{B}} A^{( \pm)}(L),
$$

if parameter $B$ is not too small; the dependence $A^{ \pm}(L)$ has been determined above, Eq. (25). The values $E_{1}^{(a)}$ and $E_{2}^{(a)}$ [Eq. (27)] coincide with $E_{1,2}$ and $E_{3,4}$, respectively, when $\mathbf{n}_{+} \cdot \mathbf{n}_{-}=-1$ in Eqs. (23) and (24).

It is important to notice that, in view of our further analysis, the various energies are located along the energy axis according to the relations $E_{1}^{(f)}>E_{1}^{(a)}>E_{2}^{(f)}>E_{3}^{(f)}>E_{2}^{(a)}>E_{4}^{(f)}$, as it can be seen from Fig. 1.

\section{CONFINEMENT-MEDIATED INTERLAYER EXCHANGE COUPLING}

The DOS of a two-dimensional parabolic band $\varepsilon\left(\mathbf{k}_{\|}\right)$ $=\mathbf{k}_{\|}^{2} / 2 m_{\|}$is equal to zero at $\omega<0$ and independent of the frequency at $\omega>0$, i.e. is $N_{2}(\omega)=N_{2} h(\omega)$, where $N_{2}$ $=m_{\|} a_{\|}^{2} /(2 \pi)$ and $h(\omega)$ is the Heaviside unit step function: $h(\omega>0)=1$ and $h(\omega<0)=0$. In the model under consideration, the DOS inside the bulk band gap, $\delta N(\omega)$, has the form of a staircaselike function,

$$
\delta N(\omega)=N_{2} \sum_{i} h\left(\omega-\varepsilon_{i}\right)
$$

where the sum is carried over the states with $\varepsilon_{i}<0$.

The two-dimensional states that split from the bulk band continuum of the undoped SC are partly filled with quasiparticles, having been transferred from transition-metal atoms inside the $\delta$ layers or/and from the spacer. Strictly speaking, an analysis of the energies of the various magnetic configurations of the DMA for a fixed total number of quasiparticles in the system (consisting of the SC host and of the FM $\delta$ layers inserted into the host) should be considered.

However, this procedure is too complex. We restrict ourselves to consider the collinear alignment of the moments $\mathbf{M}( \pm l)$.

First, we focus on the situation when the number of quasiparticles in $\delta$ layer, $n$, is strictly fixed, i.e., we assume that both the $\delta$ layer and the spacer are completely electroneutral. So far as the DOS [Eq. (28)] is known, one can write the value $n$ as

$$
\begin{aligned}
n & =N_{2} \sum_{i=1}^{4}\left[\mu^{(f)}-\varepsilon_{i}^{(f)}\right] h\left[\mu^{(f)}-\varepsilon_{i}^{(f)}\right] \\
& =2 N_{2} \sum_{i=1}^{2}\left[\mu^{(a)}-\varepsilon_{i}^{(a)}\right] h\left[\mu^{(a)}-\varepsilon_{i}^{(a)}\right],
\end{aligned}
$$

where $\mu^{(f)}$ and $\mu^{(a)}$ are the Fermi-level positions for the FM and for the AFM alignment of the moments, respectively. The corresponding expressions for the excess free energy $\delta F$ are given by

$$
\delta F^{(f)}=\frac{N_{2}}{2} \sum_{i=1}^{4}\left[\left(\mu^{(f)}\right)^{2}-\left(\varepsilon_{i}^{(f)}\right)^{2}\right] h\left(\mu^{(f)}-\varepsilon_{i}^{(f)}\right),
$$

and 


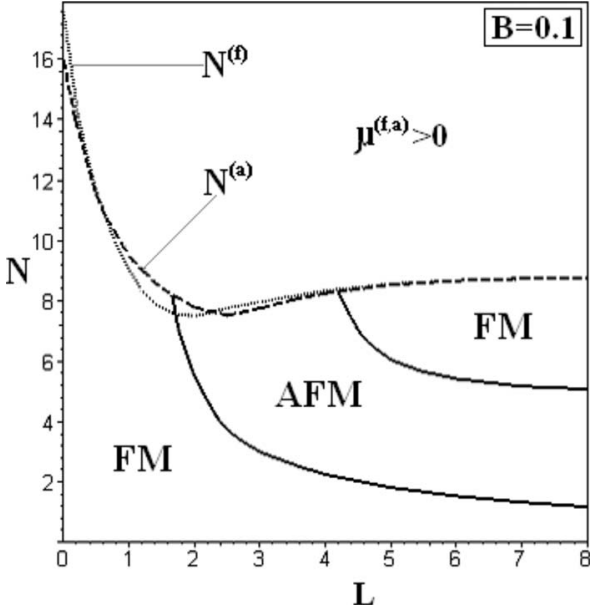

FIG. 2. The phase diagram of DMA at fixed number of quasiparticles in the $\delta$ layers, $n$ : FM and AFM regions are separated by solid curves $N(L)$ along which the exchange integral vanishes, $I_{c}(N, L)=0$; the Fermi level $\mu^{(f, a)}$ enters the conduction band above the dotted line $N^{(f)}=N\left(\mu^{(f)}=0\right)$ and the dashed line $N^{(a)}=N\left(\mu^{(a)}\right.$ $=0)$, respectively. The values of $l$ and $n$ are measured in the dimensionless units, $L=4 l m a_{z}|U|$ and $N=4 n /\left(m N_{2} a_{z}^{2} U^{2}\right)$, respectively; $B$ $=0.1$.

$$
\delta F^{(a)}=N_{2} \sum_{i=1}^{2}\left\{\left[\mu^{(a)}\right]^{2}-\left[\varepsilon_{i}^{(a)}\right]^{2}\right\} h\left[\mu^{(a)}-\varepsilon_{i}^{(a)}\right] .
$$

Excluding the values $\mu^{(f)}$ and $\mu^{(a)}$ from these expressions by means of Eq. (29), one can obtain the confinement-mediated exchange integral

$$
I_{c}=\delta F^{(f)}-\delta F^{(a)},
$$

as a function of $n$. For example, if the value of $n$ is such that only the lowest subband $\omega_{1}\left(\mathbf{k}_{\|}\right)$is partly filled, we obtain the exchange integral

$$
I_{c}=\delta F^{(f)}=\frac{n}{2}\left[\frac{n}{N_{2}}+2 \varepsilon_{1}^{(f)}\right],
$$

which is certainly FM. If the value of $n$ is such that the two-dimensional subbands $\omega_{12}^{(f)}$ and $\omega_{1}^{(a)}$ can be partly filled, and all the other subbands, $\omega_{3,4}^{(f)}$ and $\omega_{2}^{(a)}$, are empty, we obtain

$$
I_{c}=\frac{n}{2}\left[\varepsilon_{1}^{(f)}+\varepsilon_{2}^{(f)}\right]-\frac{N_{2}}{4}\left[\varepsilon_{1}^{(f)}-\varepsilon_{2}^{(f)}\right]^{2}-n \varepsilon_{1}^{(a)} .
$$

The explicit expressions for the exchange integral $I_{c}[\mathrm{Eq}$. (32)] as a function of $n$ and $l$ could be written for arbitrary occupation of the two-dimensional subbands, $\omega_{i}^{(f)}$ and $\omega_{i}^{(a)}$, with carriers. The analysis of these expressions shows that the IEC not only varies in magnitude but also switches, with varying $n$ and $l$, between being FM and AFM. Figure 2 shows that the exchange interaction at short interlayer distance and small number of electrons within the $\delta$ layer is FM. On the other hand, with increasing either $n$ or $l$ (at given $B)$, the exchange integral can change its sign up to two times. In the region $\mu^{(f, a)}>0$, our approach is not valid.
Second, let us analyze the opposite case when the Fermi level, $\mu$, is strictly fixed due to its pinning by a quasiparticle reservoir external to the $\delta$ layers. For example, the pinning could be caused by either the large DOS peak of other bulk bands (which are not explicitly considered above) or by an external electric field. In this case, we need to write the excess of thermodynamical potential: $\delta \Omega=\delta F-\mu n$. The determination of the IEC integral is read as the difference

$$
I_{c}=\delta \Omega^{(f)}-\delta \Omega^{(a)},
$$

where specific expressions for $\delta \Omega$ are given by the following formula for FM and AFM alignments of the moments $\mathbf{M}( \pm l)$, respectively:

$$
\delta \Omega^{(f)}=-\frac{N_{2}}{2} \sum_{i=1}^{4}\left(\varepsilon_{i}^{(f)}-\mu\right)^{2},
$$

and

$$
\delta \Omega^{(a)}=-N_{2} \sum_{i=1}^{2}\left(\varepsilon_{i}^{(a)}-\mu\right)^{2} .
$$

The states that lay below the Fermi level give rise to the integrals (36) and (37); in turn the position of the Fermi level is assumed to be below the bottom of the conducting band, $\mu<0$.

The following situations can occur, depending on the Fermi-level position: If $\mu$ lies inside the band gap as deep as $\varepsilon_{1}^{(f)}>\mu$, one has $\delta \Omega=0$. If the lowest level is only filled, $\varepsilon_{1}^{(a)}>\mu>\varepsilon_{1}^{(f)}$, the state with parallel moments of adjacent $\delta$ layers is realized:

$$
I_{c}=\delta \Omega^{(f)}=-N_{2}\left(\varepsilon_{1}^{(f)}-\mu\right)^{2} / 2 .
$$

However, when $\varepsilon_{1}^{(a)}<\mu$, the determination of the magnetic configuration of the system becomes cumbersome and more detailed study is necessary to clarify it. The analysis of Eq. (35) shows that, for a given $B$, IEC mediated by the confinement states changes its character from FM to AFM and vice versa with increasing interlayer distance or with the Fermi energy. The curves $\mu(l)$ along which the exchange integral vanishes, $I_{c}(\mu, l)=0$, are represented in the phase diagram of Fig. 3.

An asymptotic behavior of the confinement-mediated IEC magnitude at large value of the spacer thickness is given by the expression: $I_{c}^{( \pm)}(l) \sim \exp \left(-2 l / \xi^{( \pm)}\right)$, where the characteristic length

$$
\xi^{( \pm)}=\left[2 m a_{z}(|U| \pm|J M|)\right]^{-1}
$$

is the scale of the coupling decay in dimensional units, which is the choice between plus or minus depends on the filling of the two-dimensional subbands. The value $\xi^{( \pm)}$will be estimated in the Appendix.

\section{INTERBAND CONTRIBUTION INTO INTERLAYER EXCHANGE COUPLING}

The discussion on IEC in DMA should not be limited to the role of the confinement-induced two-dimensional subbands because other SC states exist, which can mediate an 


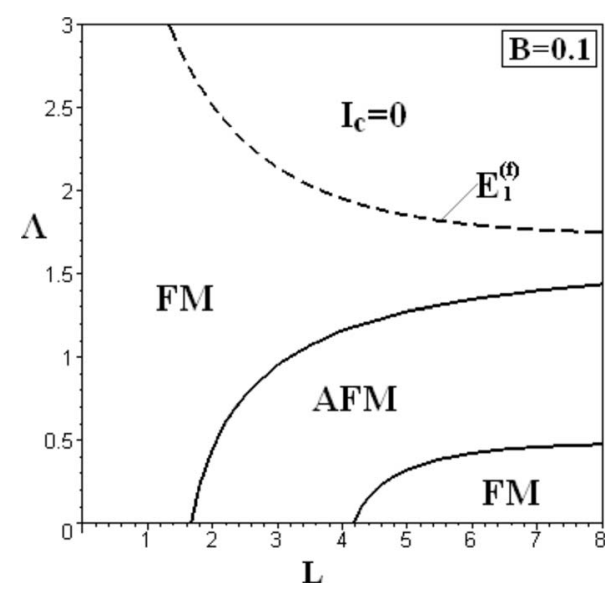

FIG. 3. The phase diagram of DMA at fixed Fermi level: the FM and AFM regions are separated by solid curves $\Lambda(L)$ along which exchange integral vanishes, $I_{c}(\Lambda, L)=0$; the coupling is absent above the dashed line $E_{1}^{(f)}$. The values of $l$ and $\mu$ are measured in the dimensionless units, $L=4 l m a_{z}|U|$ and $\Lambda=2|\mu| /\left(m a_{z}^{2} U^{2}\right)$, respectively; $B=0.1$.

effective exchange to no lesser extent. In order to allow for these processes, we now take into account the quasiparticle excitations between the bulk conduction bands and the valence bands.

In principle, one could write a general expression for the two-particle Green function, taking into account both intraband $\left(n=n^{\prime}\right)$ and interband $\left(n \neq n^{\prime}\right)$ scattering processes due to the bilayer potential in the Hamiltonian (1)-(3) associated with an appearance of deep states below the band edge. Without losing the essential physical content, we restrict ourselves to the second order in perturbation theory with respect to the dimensionless parameters that characterize the interband interaction: $\left|U_{n, n^{\prime}} g(l)\right| \ll 1$ and $\left|J_{n, n^{\prime}} g(l)\right| \ll 1$, where $n$ $\neq n^{\prime}$ and $g(l)$ is an exponentially decreasing function of the interlayer distance [Eq. (13)]. Within such an approach, one may separate intraband processes from interband processes and reduce the expression for the interband component of the effective coupling between two FM $\delta$ layers through an undoped SC spacer to a treatable form,

$$
\begin{aligned}
I_{g}(l)= & \sum_{n \neq n^{\prime}} \sum_{k_{z}} \sum_{k_{z}^{\prime}} \sum_{\mathbf{k}_{\|}} \sum_{\mathbf{k}_{\|}^{\prime}} \exp \left[i\left(k_{z}-k_{z}^{\prime}\right) 2 l\right] \\
& \times \oint \frac{d \omega}{2 \pi i} \Upsilon_{n, n^{\prime}}^{2}\left(\mathbf{k}_{\|}-\mathbf{k}_{\|}^{\prime}, \omega\right) M^{2} G_{n}^{0}(\mathbf{k}, \omega) G_{n^{\prime}}^{0}\left(\mathbf{k}^{\prime}, \omega\right),
\end{aligned}
$$

where

$$
\begin{aligned}
Y_{n, n^{\prime}}\left(\mathbf{k}_{\|}-\mathbf{k}_{\|}^{\prime}, \omega\right)= & J_{n, n^{\prime}}\left(\mathbf{k}_{\|}-\mathbf{k}_{\|}^{\prime}\right)+\frac{g_{0}(\omega)}{D(\omega)}\left[K(\omega) J_{n, n^{\prime}}\left(\mathbf{k}_{\|}-\mathbf{k}_{\|}^{\prime}\right)\right. \\
& \left.+J U_{n, n^{\prime}}\left(\mathbf{k}_{\|}-\mathbf{k}_{\|}^{\prime}\right)\right]
\end{aligned}
$$

is the renormalized interband vertex.

The intraband exchange constant $J$, and the values of $K(\omega), D(\omega)$, and $g_{0}(\omega)$ have been determined above by Eqs. (11), (10), and (12). The indices $n$ and $n^{\prime}$ refer to conduction and valence bands, respectively. According to Eq. (41), which holds provided that the interband matrix elements are comparably small, $\left|\tilde{U}_{n, n^{\prime}}\right| \ll|U|$ and $\left|\tilde{J}_{n, n^{\prime}} \ll\right| J \mid$, and the intraband interaction does not split any state from the valence band, the bare interband vertex function undergoes a renormalization, $J_{n, n^{\prime}} \rightarrow \Upsilon_{n, n^{\prime}}(\omega)$, due to the electron confinement at the metallic layers. The vertex function $Y_{n, n^{\prime}}(\omega)$ depends on the frequency $\omega$ and has singularities determined by Eq. (14). Notice that the $Y_{n, n^{\prime}}$ does not vanish even if the bare interband exchange is absent, $J_{n, n^{\prime}}=0$, but $U_{n, n^{\prime}} \neq 0$.

Properly speaking, the interband IEC is determined by the poles of the electron and hole Green's functions in the integral (40). It is clear that, at least at $2 l \gg a$, quasimomenta close to the extremal points of the band spectrum give the main contribution to the integral in Eq. (40). Therefore, in order to estimate its behavior, we can use, for a vertex function slowly varying with $\mathbf{k}$ and $\mathbf{k}^{\prime}$, the approximate expression $\Upsilon_{n, n^{\prime}}\left(\mathbf{k}_{\|}-\mathbf{k}_{\|}^{\prime}, \omega\right) \cong \Upsilon_{n, n^{\prime}}\left(\mathbf{k}_{\|}-\mathbf{k}_{\|}^{\prime}, \omega=E_{g}^{n, n^{\prime}}\right)$ $\cong Y_{n, n^{\prime}} \delta_{\mathbf{k}_{\|}-\mathbf{k}_{\|}^{\prime}, \mathbf{K}_{n, n^{\prime} \|}}$, where $E_{g}^{n, n^{\prime}}$ is the energy distance between the $n$th conduction-band minimum and the $n^{\prime}$ th valence-band maximum, which are separated by the wave vector $\mathbf{K}_{n, n^{\prime}}$ belonging to the first Brillouin zone, and $\mathbf{K}_{n, n^{\prime} \|}$ is the projection of vector $\mathbf{K}_{n, n^{\prime}}$ onto the $(x, y)$ plane. In such a case, the exchange integral can be expressed as

$$
I_{g}(l)=\sum_{n, n^{\prime}} \sum_{k_{z}} \sum_{k_{z}^{\prime}} \sum_{\mathbf{k}_{\|}} \frac{\Upsilon_{n, n^{\prime}}^{2} M^{2} \exp \left[i\left(k_{z}-k_{z}^{\prime}\right) 2 l\right]}{\varepsilon_{n \mathbf{k}}-\varepsilon_{n^{\prime} \mathbf{k}^{\prime}}},
$$

where we assume that the conduction band $(n)$ is almost empty and the valence band $\left(n^{\prime}\right)$ is almost full.

Below, we analyze the structure of the IEC integral $I_{g}(l)$ [Eq. (42)] for systems with various type of electron spectrum of the SC spacer, taking into account the effects of crystal symmetry. General expressions of the indirect exchange integral, $I_{g}(l)$, for band energy spectra of some SC (GaAs, Si, and $\mathrm{Ge}$ ) will be obtained, and then, in the Appendix, the corresponding parameters of the IEC will be estimated.

\section{A. III-V compounds electron spectrum}

In view of applications to III-V zinc blend compounds, a $\mathrm{SC}$ with a direct gap between the valence and the conduction bands at the $\Gamma$ point of the Brillouin zone should be discussed. Of course, direct interband single-particle excitations at this point provide the dominant contribution to the magnetic coupling across the intrinsic spacer in the $($ III-V)- $\delta$-doped transition-metal systems. At zero temperature, Eq. (42) describes the corresponding contribution to IEC, $I_{g}=I_{g}^{(0)}$. Assuming that the energy gap of the SC is small in comparison with the bandwidths, $W_{v} \sim W_{c} \gg E_{g}$, one can use the effective-mass description by expanding the corresponding energies around the $\Gamma$ point at $\mathbf{k}=0$,

$$
\varepsilon_{n^{\prime} \mathbf{k}^{\prime}}=-E_{g}-\frac{\left(\mathbf{k}^{\prime}\right)^{2}}{2 m_{v}},
$$




$$
\varepsilon_{n \mathbf{k}}=\frac{\mathbf{k}^{2}}{2 m_{c}^{(0)}},
$$

where $E_{g}=E_{g}^{(0)}$ is the direct-gap energy, and $m_{v}$ and $m_{c}^{(0)}$ are the effective masses of light holes and electrons, respectively. Inserting the expressions (43) and (44) into Eq. (42), it is not difficult to estimate the IEC dependence on the spacer thickness under the condition $l \gg a$,

$$
I_{g}^{(0)}(l)=-\frac{2\left[Y^{(0)}\right]^{2} M^{2} a^{4} \mu_{0}}{(4 \pi l)^{3 / 2}} \frac{\exp \left(-2 l / \zeta_{0}\right)}{\sqrt{\zeta_{0}}},
$$

where

$$
\begin{gathered}
\zeta_{0}=\left[2\left(m_{c}^{(0)}+m_{v}\right) E_{g}^{(0)}\right]^{-1 / 2}, \\
\mu_{0}=\frac{\left(m_{v} m_{c}^{(0)}\right)^{3 / 2}}{\left(m_{v}+m_{c}^{(0)}\right)^{2}},
\end{gathered}
$$

$\Upsilon^{(0)}$ is the vertex [Eq. (41)] evaluated at the wave vector $\mathbf{k}_{\|}-\mathbf{k}_{\|}^{\prime}=0$ and at the frequency $\omega=E_{g}^{(0)}$.

\section{B. Silicon electron spectrum}

In the case of the transition-metal $\delta$-doped group-IV SC host, the situation appears to be more complex. The silicon spectrum has the valence-band maximum at the point $\Gamma$ and six equivalent minima of the conduction band, located near the points $X$ of the first Brillouin zone of the diamond reciprocal lattice. The maximum of the valence band and minima of the conduction band are separated in the reciprocal space by wave vectors forming the irreducible star $\left\{\mathbf{K}_{\Gamma \chi}\right\}$ with components: $\mathbf{K}_{ \pm 1}=\frac{\pi}{2 a}(1 \pm \delta, 0,0), \mathbf{K}_{ \pm 2}=\frac{\pi}{2 a}(0,1 \pm \delta, 0)$, and $\mathbf{K}_{ \pm 3}=\frac{\pi}{2 a}(0,0,1 \pm \delta)$, where $\delta \ll 1$ and $a$ is the intermonolayer distance in the silicon crystal lattice. ${ }^{26}$

As noted above, we are considering the case of an undoped SC in which the Fermi level $\mu$ is fixed inside the indirect energy gap and Eq. (42) describes the contribution of the interband carrier excitations to IEC. We look for the poles closest to the real axis since they correspond to the longest-range magnetic coupling. It is obvious that such a singularity in the integral (42) arises from the indirect band gap having the smallest energy difference $\left|\varepsilon_{n \mathbf{k}}-\varepsilon_{n^{\prime} \mathbf{k}^{\prime}}\right|=E_{g}$ at $\mathbf{k}=\mathbf{K}_{n, n^{\prime}}=\mathbf{K}_{n}=\mathbf{K}_{\Gamma X}$ and $\mathbf{k}^{\prime}=0$. In the effective-mass approach, the conduction-band spectrum is written in the form

$$
\varepsilon_{n \mathbf{k}}=\frac{\left(\mathbf{k}_{l}-\mathbf{K}_{n}\right)^{2}}{2 m_{l}}+\frac{\mathbf{k}_{t}^{2}}{2 m_{t}},
$$

and one can use Eq. (43) to describe the valence band, where now $E_{g}=E_{g}^{(\Gamma X)}$ is the energy distance between the conduction-band minimum at the $X$ point and the valenceband [Eq. (43)] maximum at the $\Gamma$ point.

Here, it is implied that the index $n$ runs through $\pm 1, \pm 2$, and \pm 3 , assigning six conduction valleys; the single valence band has the index $n^{\prime}$, which may be omitted, and $m_{v}$ is effective mass of light holes. The dependence [Eq. (47)] has been expressed along the main axes; $m_{l}$ is longitudinal electron mass and $m_{t}$ is transverse one. After the transformations $k_{z}^{\prime}-k_{z}=q-K_{n z}$ and $\sqrt{m_{c} / m_{v}} k_{z}^{\prime}+\sqrt{m_{v} / m_{c}}\left(k_{z}-K_{n z}\right)=K$, where $m_{c}=m_{t}$ for $n= \pm 1$ and \pm 2 , and $m_{c}=m_{l}$ for $n= \pm 3$, one can rewrite the exchange integral (42) in the form

$$
\begin{aligned}
I_{g}^{(\Gamma X)}(l)= & -\sum_{n} \int \frac{d^{2} \mathbf{k}_{\|}}{(2 \pi)^{2}} \frac{d q}{2 \pi} \frac{d K}{2 \pi} \\
& \times \frac{\left(\Upsilon^{(\Gamma X)}\right)^{2} M^{2} a^{4}}{\sqrt{\frac{m_{c}}{m_{v}}}+\sqrt{\frac{m_{v}}{m_{c}}}} \frac{\exp (i 2 q l) \exp \left(-i 2 K_{n z} l\right)}{\frac{K^{2}+q^{2}}{2\left(m_{v}+m_{c}\right)}+\Lambda\left(\mathbf{k}_{\|}\right)},
\end{aligned}
$$

where

$$
\Lambda\left(k_{\|}\right)=E_{g}+\frac{\mathbf{k}_{\|}^{2}}{2}\left(\frac{1}{m_{v}}+\frac{1}{m_{c}}\right),
$$

$\mathbf{K}_{n}=\left(\mathbf{K}_{n \|}, K_{n z}\right), k_{z}$ is the projection of the vector $\mathbf{k}$ along the $\mathbf{z}$ axis, $\mathbf{k}_{\|}$is the projection of the vector $\mathbf{k}$ on the interface $(x, y)$ plane, $\Upsilon^{(\Gamma X)}$ is the vertex [Eq. (41)] evaluated at the wave vector $\mathbf{k}_{\|}-\mathbf{k}_{\|}^{\prime}=\mathbf{K}_{n \|} \neq 0$, and at the frequency $\omega=E_{g}^{(\Gamma X)}$. We skip the long calculation of the integral (48), which has already been carried out in Refs. 27 and 28, and give the final result. The asymptotical behavior of $I_{g}^{(\Gamma X)}(l)$, under condition $l \gg a$, may be evaluated as

$$
\begin{aligned}
I_{g}^{(\Gamma X)}(l)= & -\frac{2\left(\Upsilon^{(\Gamma X)}\right)^{2} M^{2} a^{4}}{(4 \pi l)^{3 / 2}}\left[2 \mu_{1} \frac{\exp \left(-2 l / \zeta_{1}\right)}{\sqrt{\zeta_{1}}}\right. \\
& \left.+\mu_{2} \frac{\exp \left(-2 l / \zeta_{2}\right)}{\sqrt{\zeta_{2}}} \cos \left(\frac{\pi l}{a}\right) \cos \left(\frac{\pi \delta l}{a}\right)\right],
\end{aligned}
$$

where

$$
\zeta_{1}=\left[2\left(m_{t}+m_{v}\right) E_{g}^{(\Gamma X)}\right]^{-1 / 2},
$$

and

$$
\begin{gathered}
\zeta_{2}=\left[2\left(m_{l}+m_{v}\right) E_{g}^{(\Gamma X)}\right]^{-1 / 2}, \\
\mu_{1}=\frac{\left(m_{v} m_{t}\right)^{3 / 2}}{\left(m_{v}+m_{t}\right)^{2}}, \\
\mu_{2}=\frac{\left(m_{v} m_{l}\right)^{3 / 2}}{\left(m_{v}+m_{l}\right)^{2}} .
\end{gathered}
$$

This expression gives the energy of the effective exchange coupling due to electron transitions with a momentum transfer $\mathbf{K}_{n}$. One sees that there exist two contributions coming from electron valleys of the SC band structure with different orientations with respect to the $\mathbf{z}$ direction. The FM biasing component, associated with the first term within the square brackets of Eq. (49), decreases exponentially with increasing spacer thickness on a length scale $\zeta_{1}$ [Eq. (50)]. The other component drops on a length scale $\zeta_{2}$ [Eq. (51)] and simultaneously oscillates from FM to AFM with two incommensurate periods as the spacer thickness $2 l$ increases. The lengths $d_{s}=4 a$ and $d_{l}=4 a / \delta$ are short-wave and long-wave periods, respectively.

Generally speaking, apart from the processes considered above with finite quasimomentum transfer, electron transitions without quasimomentum transfer at the point $\mathbf{k}=0$ could give an important contribution to IEC despite the fact that their excitation energy $E_{g}^{(0)}$ exceeds the minimal band 
gap $E_{g}^{(\Gamma X)}$ for the silicon band structure. This is due to the difference between the effective electron mass at the point $\Gamma$ and $m_{c}^{(0)}$, and at the point $X$ and $m_{l, t}$.

Combining the expressions (49) and (45), one obtains the full energy of IEC across the intrinsic silicon-type SC spacer at zero temperature, $I_{g}=I_{g}^{(0)}+I_{g}^{(\Gamma X)}$.

\section{Germanium electron spectrum}

In the conduction band of germanium, there exist eight equivalent energy minima, lying at a boundary of the Brillouin zone, at the points $L$. The maximum of valence band and minima of conduction band are separated by the wave vectors forming the irreducible star $\left\{\mathbf{K}_{\Gamma L}\right\}$ with components: $\mathbf{K}_{n}=\frac{\pi}{4 a}( \pm 1, \pm 1, \pm 1)$, where $1 \leq n \leq 8$ and $a$ is the intermonolayer distance. ${ }^{26}$ The expression for the spectrum can still be described by Eqs. (43) and (47), where now $E_{g}$ $=E_{g}^{(\Gamma L)}$ is the energy distance between the conduction-band minimum at the $L$ point and the valence-band maximum at the $\Gamma$ point. The index $n$ assigns eight conduction valleys; the single valence band has the index $n^{\prime}=0$. The calculation of the exchange integral (42) for the Ge spacer is carried out as in the case of the $\mathrm{Si}$ spacer. The coupling energy due to indirect electron transitions can be cast in the

$$
\begin{aligned}
I_{g}^{(\Gamma L)}(l)= & -\frac{2\left[\Upsilon^{(\Gamma L)}\right]^{2} M^{2} a^{4} \mu_{3}}{(4 \pi l)^{3 / 2}} \frac{\exp \left(-2 l / \zeta_{3}\right)}{\sqrt{\zeta_{3}}} \\
& \times \cos \left[\frac{3\left(m_{t}+m_{v}\right)}{m_{t}+2 m_{l}+3 m_{v}} \frac{\pi l}{2 a}\right],
\end{aligned}
$$

where

$$
\begin{gathered}
\zeta_{3}=\left[2\left(m_{m}+m_{v}\right) E_{g}^{(\Gamma L)}\right]^{-1 / 2}, \\
\frac{1}{m_{m}}=\frac{1}{3}\left(\frac{1}{m_{l}}+\frac{2}{m_{t}}\right), \\
\mu_{3}=\frac{\left(m_{v} m_{m}\right)^{1 / 2}}{m_{v}+m_{m}}\left(\frac{1}{m_{v}}+\frac{1}{m_{t}}\right)^{-1 / 2}\left(\frac{1}{m_{v}}+\frac{3}{m_{t}+2 m_{l}}\right)^{-1 / 2},
\end{gathered}
$$

$\Upsilon^{(\Gamma L)}$ is the vertex [Eq. (41)] evaluated at the vector $\mathbf{K}_{\Gamma L}$, and at the frequency $\omega=E_{g}^{(\Gamma L)}$.

The dependence $I_{g}^{\left(\mathrm{P}^{L}\right)}(l)$ on the spacer thickness consists of a factor that falls exponentially on a length scale $\zeta_{3}[\mathrm{Eq}$. (53)], modulated by an oscillatory part that changes sign from FM to AFM with a period $d_{3}=(8 / 3) a\left(m_{t}+2 m_{l}\right.$ $\left.+3 m_{v}\right) /\left(m_{t}+m_{v}\right)$. Since each electron valley located at the point $L$ of the Brillouin zone has the same projection along the $\mathbf{z}$ axis, the exchange integral $I_{g}^{(\Gamma L)}(l)[\mathrm{Eq} .(52)]$ is not divided into different components unlike the of the silicon spacer discussed above.

The full energy of IEC across the undoped germaniumtype SC spacer at zero temperature is again the sum of processes with and without quasimomentum transfer, $I_{g}=I_{g}^{(0)}$ $+I_{g}^{(\Gamma L)}$.

\section{CONCLUSIONS}

In this work, we have discussed possible mechanisms of indirect exchange between FM $\delta$ layers in DMA, taking into account the role of carrier confinement at these layers. We have shown that an efficient IEC can even be mediated by the undoped SC spacer due to virtual excitations across the energy threshold. We established the important role of quasitwo-dimensional spin-polarized states inside the bulk energy gap, which are caused by confinement. Quasiparticle excitations from these states to the band edge significantly contribute to the interlayer coupling; the magnitude and character of which strongly depend on the parameters of the DMA. Varying the filling of the confinement states or the spacer thickness, one could switch the exchange coupling between FM and AFM. Taking into account effects of crystal symmetry, we have also obtained the expression for the interband coupling energy in the case of both direct and indirect-gap spacers. The interband coupling decays exponentially with the distance between the $\delta$ layers and is strongly dependent on the electron structure of the host. The combination of the two mechanisms - confinement-mediated exchange and interband exchange-mainly determines the behavior of IEC in DMA with undoped spacer.

Main assumptions of our model and the results obtained are backed by more rigorous ab initio calculations. In particular, a two-dimensional ferromagnetic half-metallic character of DMA was found by calculations in GaAs deltadoped with $\mathrm{Mn} .{ }^{15}$ It was shown that the in-plane conductivity is metallic (only for spin-up) due to hole carriers confined at and close to the Mn layers while the conductivity perpendicular to the plane is extremely low, reflecting the tunneling between the Mn layers. Calculations on a delta-doped layer of $\mathrm{Mn}$ in Si (Refs. 21 and 22) and Ge (Refs. 19 and 20) also demonstrated two-dimensional half-metallic ferromagnetism. Furthermore, the magnetic coupling between two Mn-doped layers as a function of number of Ge spacing layers for different concentrations of manganese was studied within density-functional theory. ${ }^{19}$ All these systems showed FM as the most stable magnetic alignment except for the structure that consists of the three Ge spacer atomic layers and full Mn monolayer: the latter exhibits the AFM state. As the number of $\mathrm{Ge}$ atomic layers increases, the value of IEC decreases, becoming less than $0.01 \mathrm{eV}$ (close to the limit of numerical accuracy ${ }^{19}$ ) at five Ge spacer atomic layers. In $a b$ initio study $^{29}$ of the carrier-induced magnetization reversal in digital $(\mathrm{Ga}, \mathrm{Mn})$ As heterostructures with varying distance between two Mn monolayers along with the distribution and concentration of external carriers, it was found that the presence of external holes switches the AFM state to FM one when the interlayer Mn-Mn distance is of $16.96 \AA$, whereas the addition of electrons produced no significant impact. This effect was referred to the spin polarization of the SC spacer states and half-metallic feature of the system.

The discussed findings of first-principles simulations ${ }^{19,29}$ qualitatively match our results: magnetic interlayer interaction in DMA with undoped spacer is strictly coupled with the confined (localized) spin-polarized carriers and seems to have the same spatial extension. A sign change in IEC can take place as the spacer thickness or the filling of the con- 
finement states vary. It is believed that the common peculiarities of the magnetic phase diagram of DMA (Figs. 2 and 3 ), i.e., the switching between FM and AFM phases, could be reproduced within computational approaches. And what is more, under a high numerical accuracy condition, the IEC dependence on the DMA period can be described, taking into account a self-consistent redistribution of both charge and spin densities.

Let us finally discuss some unsolved theoretical problems of DMA. In our opinion, the most important task is to develop the spin-fluctuation theory of itinerant magnetism for a transition-metal $\delta$ layer inserted into a SC host. On the other hand, in the attempt to apply our model to real situation in DMA, we are faced with the following difficult questions. What kind of magnetic order can occur in a strongly inhomogeneous quasi-two-dimensional layer? How can one describe IEC in DMA with a dirty spacer of nondegenerate SC containing impurities and clusters? In order to answer these questions, it is necessary to include in the model the impurity scattering effects.

It is also desirable to extend our treatment to other DMA, which are different from those usually studied, i.e., $\mathrm{GaAs} / \mathrm{Mn}$ and Ge/Mn, both with respect to the SC host [for instance, $\mathrm{GaSb}, \mathrm{GaN},(\mathrm{Ga}, \mathrm{Al}) \mathrm{As}$, and binary alloys of type $\left.\mathrm{Si}_{x} \mathrm{Ge}_{1-x}\right]$ and with respect to the metal insertion (for instance, $\mathrm{Fe}, \mathrm{Co}, \mathrm{Cr}$, and their alloys). Moreover, it would be crucial to carry out a comparative analysis of the magnetic properties of DMA with various morphologies and orientations of the $\delta$ layers when transition-metal ions could occupy substitutional or interstitial positions in planes with crystalline indices such as (001), (011), and (111).

As a concluding remark, we point out another result that can be proved without going far beyond the scope of our scheme to treat the confinement-mediated exchange. It could be shown that any noncollinear state for the system under consideration, $\mathbf{n}_{+} \cdot \mathbf{n}_{-} \neq \pm 1$, is always energetically unfavorable with respect to the two collinear states, either the FM, $\mathbf{n}_{+} \cdot \mathbf{n}_{-}=1$, or the AFM, $\mathbf{n}_{+} \cdot \mathbf{n}_{-}=-1$. It is necessary to point that if one takes into account the imperfection of the $\delta$ layer associated to its roughness, the noncollinear alignment of the moments could become favorable at some nominal spacer thicknesses near a critical value $L^{*}(B)$. In this case, the magnetic phase diagram of DMA could acquire the regions of noncollinear states along FM-AFM boundaries in Figs. 2 and 3. The roughness effect can be treated, for instance, in the framework of the model of Ref. 30.

\section{ACKNOWLEDGMENTS}

The work was partially supported by the University of the Basque Country (Grant No. 9/UPV 00206.215-13639/2001), by Spanish Ministerio de Ciencia y Tecnología (Grant No. FIS 2004-06490-C03-01), and by RFBR (Grant No. 07-0200114-a).

\section{APPENDIX}

Our simplified model describes the states induced by the FM $\delta$ layer with energies lying either below the bottom of the conduction band or above the top of the valence band. It should be emphasized that, in real DMA with complex band structure, these states are not constrained to reside in the band gap and could be deep enough to enter into the band continuum region. Although the states induced by the FM $\delta$ layer possess a clear two-dimensional character and have their wave functions confined to one or two SC monolayers adjacent to the transition-metal monolayer or submonolayer, they play an important role in the IEC due to the strong potential and exchange scattering of quasiparticles of the SC spacer on the plane of FM defects. Unfortunately, at the present, the lack of throughout experimental data does not allow us to correctly obtain the above mentioned scattering parameters. We can only give rough estimates of the characteristic lengths $\xi^{( \pm)}$based on the electron structure calculations. ${ }^{15-22}$ Extracting the local DOS shifts for the spin-up and spin-down two-dimensional bands relative to the bulk SC band, one can derive: $|U| \approx 2-3 \mathrm{eV}$ and $|J M|$ $\approx 1-2 \mathrm{eV}$. If one takes $m=0.1 m_{0}\left(m_{0}\right.$ is the free-electron mass) and $a \approx 5 \AA$, the decaying scales of the confinementmediated IEC are $\xi^{(+)} \approx 2-3 \AA$ and $\xi^{(-)} \approx 3-5 \AA$. In turn, under the same assumptions, as it can be seen from Figs. 2 and 3, the critical spacer thickness corresponding to the switching between the parallel and antiparallel configurations of adjacent FM $\delta$ layers would be of the same order as (or larger than) $\xi^{( \pm)}$.

Sufficiently reliable estimates can be obtained for the interband component of IEC. For pure crystalline GaAs, the main contribution to IEC is due to direct electron excitations through the optical gap $E_{g}^{(0)}$ at the point $\Gamma$. Using well-known data: $\quad m_{c}^{(0)}=0.065 m_{0}, \quad m_{v}=0.15 m_{0}, \quad a=1.41 \AA$, and $E_{g}^{0}$ $=1.5 \mathrm{eV},{ }^{31}$ one may derive from Eq. (46) the parameters of the exchange integral (45), $\zeta_{0}=3.44 \AA$ and $\mu_{0}=0.021 m_{0}$.

For diamondlike SC, there are two main contributions. One originates from direct electron excitations through the optical gap $E_{g}^{(0)}$ at the point $\Gamma$. The other is determined by indirect electron excitations through the thermal gap $E_{g}^{(\Gamma X)}$ for $\mathrm{Si}$ or $E_{g}^{(\Gamma L)}$ for $\mathrm{Ge}$. However, as a rule, the electron effective mass at the point $\mathbf{k}=0$ is smaller than the mass at the point $\mathbf{k}=\mathbf{K}_{n}$. Eventually, in the real case, the direct band excitations give rise to a longer-scale coupling in comparison with the indirect band excitations, $\zeta_{0}>\zeta_{1,2}$ for Si spacer and $\zeta_{0}>\zeta_{4}$ for Ge spacer, although $E_{g}^{0}>E_{g}^{(\Gamma X)}, E_{g}^{(\Gamma L)}$.

The band parameters of pure crystalline silicon can be taken from the textbook: ${ }^{31} E_{g}^{(\Gamma X)}=1.17 \mathrm{eV}, E_{g}^{(0)}=3.4 \mathrm{eV}$, $m_{l}=0.92 m_{0}, \quad m_{t}=0.19 m_{0}, \quad m_{c}^{(0)}=0.156 m_{0}, \quad m_{v}=0.16 m_{0}, \quad a$ $=1.35 \AA$, and $\delta=0.18$. Therefore the following estimates hold: $\zeta_{0}=1.89 \AA, \zeta_{1}=3.06 \AA, \zeta_{2}=1.74 \AA, \mu_{0}=0.039 m_{0}$, $\mu_{1}=0.043 m_{0}, \mu_{2}=0.048 m_{0}, d_{s} \approx 5.4 \AA$, and $d_{l} \approx 27 \AA$.

For pure crystalline germanium, it is well known that $m_{l}$ $=1.59 m_{0}, \quad m_{t}=0.082 m_{0}, \quad m_{c}^{(0)}=0.041 m_{0}, \quad m_{v}=0.04 m_{0}, \quad a$ $=1.41 \AA$, and the minimal indirect band gap is $E_{g}=E_{g}^{\Gamma L}$ $=0.744 \mathrm{eV}$, close to the value of the direct band gap, $E_{g}^{0}$ $=0.898 \mathrm{eV}$ at the point $\Gamma(\mathbf{k}=0),{ }^{31}$ so that the following estimates hold: $\zeta_{0}=7.25 \AA, \zeta_{4}=5.67 \AA, \mu_{0}=0.010 m_{0}, m_{m}$ $=0.12 m_{0}, \mu_{4}=0.0140 m_{0}$, and $d_{4}=103.8 \AA$.

We note that the two mechanisms of IEC in DMA considered above (the confinement-mediated and interband) decay over comparable length scales. 
${ }^{1}$ I. Žutić, J. Fabian, and S. Das Sarma, Rev. Mod. Phys. 76, 323 (2004).

${ }^{2}$ R. K. Kawakami, E. Johnston-Halperin, L. F. Chen, M. Hanson, N. Guébels, J. S. Speck, A. C. Gossard, and D. D. Awshalom, Appl. Phys. Lett. 77, 2379 (2000).

${ }^{3}$ T. C. Kreutz, G. Zanelatto, E. G. Gwinn, and A. C. Gossard, Appl. Phys. Lett. 81, 4766 (2002).

${ }^{4}$ H. Luo, B. D. McCombe, M. H. Na, K. Mooney, F. Lehmann, X. Chen, M. Cheon, S. M. Wang, Y. Sasaki, X. Liu, and J. K. Furdina, Physica E (Amsterdam) 12, 366 (2002).

${ }^{5}$ X. Chen, M. Na, M. Cheon, S. Wang, H. Luo, B. D. McCombe, X. Liu, Y. Sasaki, T. Wojtowicz, J. K. Furdina, S. J. Potashnik, and P. Schiffer, Appl. Phys. Lett. 81, 511 (2002).

${ }^{6}$ B. D. McCombe, M. Na, X. Chen, M. Cheon, S. M. Wang, H. Luo, X. Liu, Y. Sasaki, T. Wojtowicz, J. K. Furdina, S. J. Potashnik, and P. Schiffer, Physica E (Amsterdam) 16, 90 (2003).

${ }^{7}$ A. M. Nazmul, S. Kobayashi, S. Sugahara, and M. Tanaka, Physica E (Amsterdam) 21, 937 (2004).

${ }^{8}$ A. M. Nazmul, S. Sugahara, and M. Tanaka, Phys. Rev. B 67, 241308(R) (2003).

${ }^{9}$ Y. D. Park, A. Wilson, A. T. Hanbicki, J. E. Mattson, T. Ambrose, G. Spanos, and B. T. Jonker, Appl. Phys. Lett. 78, 2739 (2001).

${ }^{10}$ S. Cho, S. Choi, S. C. Hong, Y. Kim, J. B. Ketterson, B.-J. Kim, Y. C. Kim, and J.-H. Jung, Phys. Rev. B 66, 033303 (2002).

${ }^{11}$ H. Weng and J. Dong, Phys. Rev. B 71, 035201 (2005).

${ }^{12}$ M. Bolduc, C. Awo-Affouda, A. Stollenwerk, M. B. Huang, F. G. Ramos, G. Agnello, and V. P. LaBella, Phys. Rev. B 71, 033302 (2005).

${ }^{13}$ D. E. Bürgler, M. Buchmeier, S. Gramm, S. Eisebitt, R. R. Gareev, P. Grunberg, C. L. Jia, L. L. Pohlmann, R. Schreiber, M. Siegel, Y. L. Qin, and A. Zimina, J. Phys.: Condens. Matter 15,
S443 (2003).

${ }^{14}$ G. S. Patrin, S. G. Ovchinnikov, D. A. Velikanov, and V. P. Kononov, Phys. Solid State 43, 1712 (2001).

${ }^{15}$ S. Sanvito and N. A. Hill, Phys. Rev. Lett. 87, 267202 (2001).

${ }^{16}$ M. C. Qian, C. Y. Fong, W. E. Pickett, J. E. Pask, L. H. Yang, and S. Dag, Phys. Rev. B 71, 012414 (2005).

${ }^{17}$ M. C. Qian, C. Y. Fong, and W. E. Pickett, J. Appl. Phys. 99, 08D517 (2006).

${ }^{18}$ X. H. Zhou, X. Chen, Y. Huang, H. Duan, L. Z. Sun, and W. Lu, J. Appl. Phys. 99, 113903 (2006).

${ }^{19}$ A. Continenza, F. Antoniella, and S. Picozzi, Phys. Rev. B 70, 035310 (2004)

${ }^{20}$ H.-Y. Wang and M. C. Qian, J. Appl. Phys. 99, $08 D 705$ (2006).

${ }^{21}$ M. C. Qian, C. Y. Fong, K. Liu, W. E. Pickett, J. E. Pask, and L. H. Yang, Phys. Rev. Lett. 96, 027211 (2006).

${ }^{22}$ H. Wu, P. Kratzer, and M. Scheffler, Phys. Rev. Lett. 98, 117202 (2007).

${ }^{23}$ T. Moriya, Spin Fluctuation in Itinerant Electron Magnetism (Springer, Berlin, 1985).

${ }^{24}$ Zhu-Pei Shi, P. Levy, and J. Fry, Europhys. Lett. 26, 473 (1994).

${ }^{25}$ V. V. Tugushev, V. N. Men'shov, I. A. Nechaev, and E. V. Chulkov, Phys. Rev. B 74, 184423 (2006).

${ }^{26}$ G. L. Bir and G. E. Picus, Symmetry and Strain-Induced Effects in Semiconductors (Nauka, Moscow, 1972).

${ }^{27}$ R. Sokel and W. A. Harrison, Phys. Rev. Lett. 36, 61 (1976).

${ }^{28}$ K. Xia, W. Zhang, M. Lu, and H. Zhai, Phys. Rev. B 56, 14901 (1997).

${ }^{29}$ Jisang Hong, Ding-Sheng Wang, and R. Q. Wu, Phys. Rev. Lett. 94, 137206 (2005).

${ }^{30}$ J. C. Slonczewski, J. Magn. Magn. Mater. 150, 13 (1995).

${ }^{31}$ I. M. Tsidilkovskii, Electrons and Holes in Semiconductors (Nauka, Moscow, 1978). 